\title{
Input Impedance Modeling of Single-Phase Voltage Source Rectifier With Consideration of FrequencyCoupling Effect
}

This paper was downloaded from TechRxiv (https://www.techrxiv.org).

\section{LICENSE}

CC BY 4.0

\section{SUBMISSION DATE / POSTED DATE}

02-04-2020 / 03-04-2020

\section{CITATION}

Lin, Jianheng; Su, Mei; Sun, Yao; Xie, Shiming (2020): Input Impedance Modeling of Single-Phase Voltage Source Rectifier With Consideration of FrequencyCoupling Effect. TechRxiv. Preprint. https://doi.org/10.36227/techrxiv.12061752.v1

DOI 


\section{Input Impedance Modeling of Single-Phase Voltage Source Rectifier With Consideration of Frequency- Coupling Effect}

\author{
Jianheng Lin \\ School of Automation \\ Central South University \\ Changsha, China \\ mltortoise@163.com
}

\author{
Mei Su \\ School of Automation \\ Central South University \\ Changsha, China \\ sumeicsu@mail.csu.edu.cn
}

\author{
Shiming Xie \\ School of Automation \\ Central South University \\ Changsha, China \\ shimingxie@csu.edu.cn
}

\author{
Yao Sun \\ School of Automation \\ Central South University \\ Changsha, China \\ yaosuncsu@gmail.com
}

\begin{abstract}
Time-periodicity and non-linearity pose a challenge to the precise input impedance modeling of single-phase power converters. In this study, a precise input impedance model with measurability of the single-phase voltage source rectifier (VSR), which considers the frequency-coupling effect (FCE), is established. Meanwhile, it is revealed that the rectifier input impedance is dependent of the grid impedance. In the proposed modeling approach, only Laplace transform and frequencyshifting operation are required, which avoids the complicated convolution calculation in the frequency domain. In addition, the influence of grid impedance on the input impedance is studied. Simulations are conducted to verify the effectiveness of the proposed method.
\end{abstract}

Keywords-Input impedance, single-phase voltage source rectifier, frequency-coupling effect.

\section{INTRODUCTION}

Single-phase voltage source converters have been widely employed in power systems, such as Distributed Generation (DG), High-Voltage DC (HVDC) transmissions, and electric railway traction [1]. The power systems integrated with a large share of power electronics converters may be unstable, due to the dynamic interactions between power converters and the ac source [2]-[3]. Especially, at the Point of the Common Coupling (PCC), the harmonics excited by the presence of the grid impedance often lead to system instability [4].

Since the operation trajectory of single-phase system is not a fixed point in steady state, the conventional modeling methods based on the Linear-Time-Invariant (LTI) theory are no longer suitable [5]-[6]. The time-periodicity is one main cause of introducing Frequency-Coupling Effect (FCE) [7]-[8]. Impedance model have the merits of clear physical meaning and measurability. Therefore, impedance modeling has become the focus in dealing with the time-periodicity challenges in singlephase systems.

Generally, due to the nonexistence of orthogonal signal in single-phase system, the methods designed for three-phase systems cannot be directly applied to analyze single-phase ones. In this case, a single-phase $d q$-decomposition method without

This work is supported by the National Natural Science Foundation of China under Grant 61933011. the Park transformation is proposed [9], whereas the built model is only accurate in the regions under $15 \%$ of the fundamental frequency. In [10] the dynamic phasor approach is used to obtain the impedances, but the assumption that all state variables are dominated by fundamental-frequency components may lose some important information. Harmonic linearization is effective in developing a small-signal linear model for the non-linear time periodic systems [11], in which the overlook of frequencycoupling dynamics limits the accuracy of this method in the lowfrequency regions. In order to improve the accuracy of the model, the frequency interactions up to twice the line frequency are taken into consideration, giving a mirror-frequency model [12]. However, only the fundamental perturbed frequency harmonic in dc-side is considered. The single-frequency impedance model of single-phase inverter is established in [13], where only the PLL will result in FCE and other parts are linear. In contrast, the topology of single-phase VSR features inherent nonlinearity. And the extra dc-link controller will also lead to FCE except for the PLL. These characteristics complicate the impedance modeling of single-phase VSR.

In this paper, a single-frequency input impedance of singlephase VSR with measurability is established with consideration of the FCE. Based on the established impedance model, it is revealed that the grid impedance has a shaping effect on the rectifier input impedance, whereas the influence analysis of grid impedance is missing in most literatures. And the proposed method only requires Laplace transform and frequency-shifting operation, which eliminate the complicated convolution calculation in the frequency domain. Simulation studies are carried out to verify the validity of the proposed method.

\section{INPUT IMPEDANCE MODELING}

\section{A. System Description}

The circuit configuration and its equivalent Thevenin model of the studied single-phase VSR are depicted in Fig. 1. The main circuit is comprised of a power supply $u_{g}$, a grid impedance $L_{g} / R_{g}$, an input inductance $L$, a H-bridge circuit, an output capacitance $C_{d c}$ and a load resistance $R_{d c}$. A PLL is used to obtain the angle of the PCC voltage $u_{i}$. And the dc-link voltage $u_{d c}$ and input current $i_{g}$ are regulated by a voltage loop and a current loop. 


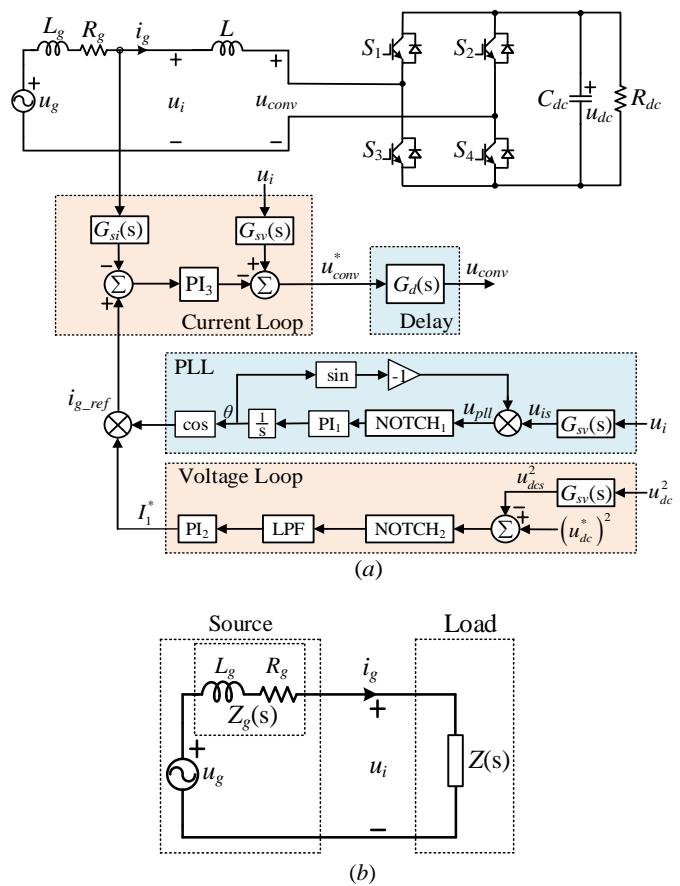

Fig. 1. (a) Control block diagram and (b) equivalent Thevenin model of singlephase voltage source rectifier.

In this system, the sampling delay is modeled as

$$
\begin{aligned}
G_{s i}(s) & =\frac{1}{1+s / \omega_{i}} \\
G_{s v}(s) & =\frac{1}{1+s / \omega_{v}}
\end{aligned}
$$

where $\omega_{i}$ and $\omega_{v}$ are the cutoff frequency of anti-aliasing filter for current and voltage measurement, respectively. In addition, a low-pass filter (LPF), two notch filters (NOTCH) and three proportional-integral (PI) controllers are given as

$$
\begin{gathered}
\operatorname{LPF}(s)=\frac{1}{1+\tau s} \\
\operatorname{NOTCH}_{m}(s)=\frac{s^{2}+\omega_{n}^{2}}{s^{2}+\sigma s+\omega_{n}^{2}} \quad(m=1,2) \\
P I_{m}(s)=k_{p m}+\frac{k_{i m}}{s} \quad(m=1,2,3)
\end{gathered}
$$

where $\tau$ is the time constant, $\sigma$ is bandwidth coefficient, $\omega_{n}$ is the center frequency, and $k_{p m}$ and $k_{i m}$ are the corresponding controller parameters. The computational delay and the delay introduced by Zero-Order Hold ( $\mathrm{ZOH})$ of the PWM are taken into consideration as

$$
G_{d}(s)=e^{-s T_{s}} \frac{1-e^{-s T_{s}}}{s T_{s}}
$$

where $T_{s}$ is the control period.

B. Modeling of the PLL

Assuming the input voltage and current in steady state are

$$
\begin{gathered}
u_{i}^{*}(t)=V_{1} \cos \left(\omega_{1} t\right) \\
i_{g}^{*}(t)=I_{1} \cos \left(\omega_{1} t+\varphi_{i 1}\right)
\end{gathered}
$$

where $V_{1}$ is the amplitude of the PCC voltage, $\omega_{1}$ is the angular frequency of the input voltage corresponding to fundamental frequency $f_{1}$, and $I_{1}$ and $\varphi_{\mathrm{i} 1}$ correspond to the magnitude and initial phase of the fundamental current, respectively.

The PLL is used to track the phase of the PCC voltage $u_{i}$ as shown in Fig. 1(a). The time-domain expression of the nonlinear part in PLL can be obtained as

$$
u_{p l l}(t)=-\sin [\theta(t)] u_{i s}(t) .
$$

By perturbing the variables in the time-domain, the linearized form of (9) is derived as

$$
\tilde{u}_{p l l}(t)=-\cos \left[\theta^{*}(t)\right] u_{i s}^{*}(t) \tilde{\theta}(t)-\sin \left[\theta^{*}(t)\right] \tilde{u}_{i s}(t)
$$

where the superscript ' $\sim$ ' denotes the small-signal quantity, and $G_{s v}(s)$ sampling at the fundamental frequency is neglected that $G_{s v}\left(j \omega_{1}\right) \approx 1$ such that $u_{i s}{ }^{*}(t)=V_{1} \cos \left(\omega_{1} t\right)$ and $\theta^{*}(t)=\omega_{1} t$.

Applying Laplace transform to (10) gives

$$
\begin{aligned}
\tilde{u}_{p l l}(s)= & -\frac{V_{1}}{2} \tilde{\theta}(s)-\frac{V_{1}}{4}\left[\tilde{\theta}\left(s-j 2 \omega_{1}\right)+\tilde{\theta}\left(s+j 2 \omega_{1}\right)\right] . \\
& +\frac{j}{2}\left[\tilde{u}_{i s}\left(s-j \omega_{1}\right)-\tilde{u}_{i s}\left(s+j \omega_{1}\right)\right]
\end{aligned}
$$

Based on the PLL diagram, the following relationships hold

$$
\begin{gathered}
\tilde{u}_{i s}(s)=G_{s v}(s) \tilde{u}_{i}(s) \\
\tilde{\theta}(s)=H_{P L L}(s) \tilde{u}_{p l l}(s)
\end{gathered}
$$

where

$$
H_{P L L}(s)=\operatorname{NOTCH}_{1}(s) \cdot P I_{1}(s) \cdot \frac{1}{s}
$$

Substituting (12) and (13) into (11), it is deduced in (15) shown at the bottom of the page, where $G_{P L L}(s)$ is

$$
G_{P L L}(s)=\frac{H_{P L L}(s)}{2+V_{1} H_{P L L}(s)} .
$$

It is worth noting that the perturbed phase $\tilde{\theta}(s)$ at a given frequency $\omega$ is related to not only the PCC voltage at the two coupled frequencies $\omega \pm \omega_{1}$ but also the perturbed phase at frequencies $\omega \pm 2 \omega_{1}$.

\section{Modeling of Voltage Loop}

According to the power balancing principle, the relationship between the input and the output can be described as

$$
u_{i}(t) i_{g}(t)=L i_{g}(t) \frac{d i_{g}(t)}{d t}+\frac{C_{d c}}{2} \frac{d x_{u}(t)}{d t}+\frac{x_{u}(t)}{R_{d c}}
$$

where $x_{u}(t)=u_{d c}{ }^{2}(t)$. Applying linearization to (17) gives (18), shown at the bottom of next page. Substituting (7) and (8) into (18) and performing Laplace transform, (19) is obtained, which is shown at the bottom of next page. And $G_{L}(s)$ is given by

$$
G_{L}(s)=\frac{R_{d c}}{C_{d c} R_{d c} s+2} .
$$

It can be seen that $\tilde{x}_{u}(s)$ is dependent on $u_{i}$ and $i_{g}$ at two coupled frequencies $\omega \pm \omega_{1}$. According to the voltage control scheme in Fig. 1(a), the small-signal term of $I_{1}{ }^{*}$ is derived as

$$
\tilde{I}_{1}^{*}(s)=H_{v}(s) \tilde{x}_{u}(s)
$$

where

$$
H_{v}(s)=-P I_{2}(s) L P F(s) N \operatorname{LTCH}_{2}(s) G_{v}(s) .
$$

$$
\tilde{\theta}(s)=G_{P L L}(s)\left\{-\frac{V_{1}}{2}\left[\tilde{\theta}\left(s-j 2 \omega_{1}\right)+\tilde{\theta}\left(s+j 2 \omega_{1}\right)\right]+j\left[G_{s v}\left(s-j \omega_{1}\right) \tilde{u}_{i}\left(s-j \omega_{1}\right)-G_{s v}\left(s+j \omega_{1}\right) \tilde{u}_{i}\left(s+j \omega_{1}\right)\right]\right\}
$$




\section{Modeling of Current Loop}

The input current reference is

$$
i_{g_{-} r e f}(t)=I_{1}^{*}(t) \cos [\theta(t)] .
$$

Then, the small-signal form of (23) is given as

$$
\tilde{i}_{g_{-r e f}}(t)=-I_{1} \sin \left[\theta^{*}(t)\right] \tilde{\theta}(t)+\cos \left[\theta^{*}(t)\right] \tilde{I}_{1}^{* \prime}(t) .
$$

Similarly, the $s$-domain expression of (24) can be deduced as

$$
\begin{aligned}
\tilde{i}_{g_{-} r e f}(s)= & \frac{j I_{1}}{2}\left[\tilde{\theta}\left(s-j \omega_{1}\right)-\tilde{\theta}\left(s+j \omega_{1}\right)\right] . \\
& +\frac{1}{2}\left[\tilde{I}_{1}^{*}\left(s-j \omega_{1}\right)+\tilde{I}_{1}^{*}\left(s+j \omega_{1}\right)\right]
\end{aligned}
$$

It can be seen from (21) and (25) that two perturbed components of output voltage at frequencies $\omega \pm \omega_{1}$ contribute to the harmonic current at frequency $\omega$.

Frequency-shifting (15) to $\omega \pm \omega_{1}$ yields (26) shown at the bottom of the page. Since the components of the perturbed phase are coupled with each other in different domains, a truncation is made that the harmonic components of perturbed phase with triple line frequency deviation are assumed to be zero. Therefore, after some mathematical manipulations, $\tilde{\theta}\left(s-j \omega_{1}\right)$ and $\tilde{\theta}\left(s+j \omega_{1}\right)$ are extracted in (27) shown at the bottom of the page. And $\Delta(\mathrm{s})$ in (27) is given as

$$
\Delta(s)=1-\frac{V_{1}^{2}}{4} G_{P L L}\left(s-j \omega_{1}\right) G_{P L L}\left(s+j \omega_{1}\right) .
$$

Then, the difference of perturbed phase at frequencies $\omega \pm \omega_{1}$ is provided as

$$
\tilde{\theta}\left(s-j \omega_{1}\right)-\tilde{\theta}\left(s+j \omega_{1}\right)=f_{\theta u,-2}(s) \tilde{u}_{i}\left(s-j 2 \omega_{1}\right)
$$$$
+f_{\theta u, 0}(s) \tilde{u}_{i}(s)+f_{\theta u, 2}(s) \tilde{u}_{i}\left(s+j 2 \omega_{1}\right)
$$

where $f_{\theta u,-2}(s), f_{\theta u, 0}(s)$ and $f_{\theta u, 2}(s)$ are given in (30) shown at the bottom of the page.

In a similar way, frequency-shifting (21) to $\omega \pm \omega_{1}$ and doing an addition yield (31) shown at the bottom of the page, where $f_{I u,-2}(s), f_{I L, 0}(s)$ and $f_{I L, 2}(s)$ are given as

$$
\begin{gathered}
f_{L u,-2}(s)=G_{L}\left(s-j \omega_{1}\right) H_{v}\left(s-j \omega_{1}\right) I_{1} e^{j \varphi_{i l}} \\
f_{l u, 0}(s)=I_{1}\left[\begin{array}{l}
G_{L}\left(s+j \omega_{1}\right) H_{v}\left(s+j \omega_{1}\right) e^{j \varphi_{i 1}} \\
+G_{L}\left(s-j \omega_{1}\right) H_{v}\left(s-j \omega_{1}\right) e^{-j \varphi_{11}}
\end{array}\right] \\
f_{I u, 2}(s)=G_{L}\left(s+j \omega_{1}\right) H_{v}\left(s+j \omega_{1}\right) I_{1} e^{-j \varphi_{11}}
\end{gathered}
$$

And $f_{i i,-2}(s), f_{i i, 0}(s)$ and $f_{l i, 2}(s)$ are given in (33) shown at the bottom of the page.

\section{E. Closed-Loop Impedance Derivation}

Based on the control scheme in Fig. 1(a), the converter voltage can be written as

$$
\begin{aligned}
\tilde{u}_{c o n v}(s)= & -G_{d}(s) P I_{3}(s) \tilde{i}_{s-r e f}(s)+G_{d}(s) G_{s v}(s) \tilde{u}_{i}(s) \\
& +G_{d}(s) P I_{3}(s) G_{s i}(s) \tilde{i}_{g}(s)
\end{aligned}
$$

And the current dynamics in the average model is

$$
L \tilde{i}_{g}(s)=\tilde{u}_{i}(s)-\tilde{u}_{\text {conv }}(s)
$$

\begin{tabular}{|c|c|}
\hline 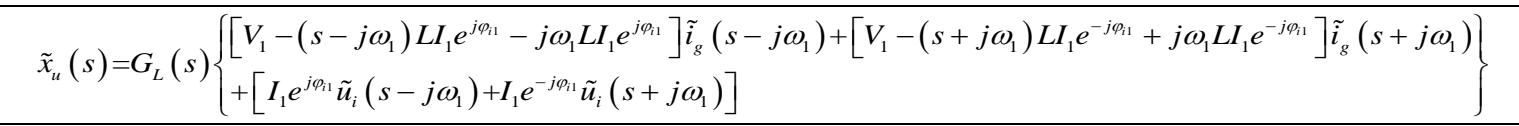 & (19) \\
\hline$\tilde{\theta}\left(s-j \omega_{1}\right)=G_{P L L}\left(s-j \omega_{1}\right)\left\{-\frac{V_{1}}{2}\left[\tilde{\theta}\left(s-j 3 \omega_{1}\right)+\tilde{\theta}\left(s+j \omega_{1}\right)\right]+j\left[G_{s v}\left(s-j 2 \omega_{1}\right) \tilde{u}_{i}\left(s-j 2 \omega_{1}\right)-G_{s v}(s) \tilde{u}_{i}(s)\right]\right\}$ & $(26 . a)$ \\
\hline$\tilde{\theta}\left(s+j \omega_{1}\right)=G_{P L L}\left(s+j \omega_{1}\right)\left\{-\frac{V_{1}}{2}\left[\tilde{\theta}\left(s-j \omega_{1}\right)+\tilde{\theta}\left(s+j 3 \omega_{1}\right)\right]+j\left[G_{s v}(s) \tilde{u}_{i}(s)-G_{s v}\left(s+j 2 \omega_{1}\right) \tilde{u}_{i}\left(s+j 2 \omega_{1}\right)\right]\right\}$ & $(26 . b)$ \\
\hline$\tilde{\theta}\left(s-j \omega_{1}\right)=\frac{j G_{P L L}\left(s-j \omega_{1}\right)}{2 \Delta(s)}\left\{\begin{array}{l}2 G_{s v}\left(s-j 2 \omega_{1}\right) \tilde{u}_{i}\left(s-j 2 \omega_{1}\right)-\left[2+V_{1} G_{P L L}\left(s+j \omega_{1}\right)\right] G_{s v}(s) \tilde{u}_{i}(s) \\
+V_{1} G_{P L L}\left(s+j \omega_{1}\right) G_{s v}\left(s+j 2 \omega_{1}\right) \tilde{u}_{i}\left(s+j 2 \omega_{1}\right)\end{array}\right\}$ & $(27 . a)$ \\
\hline$\tilde{\theta}\left(s+j \omega_{1}\right)=\frac{j G_{P L L}\left(s+j \omega_{1}\right)}{2 \Delta(s)}\left\{\begin{array}{l}-V_{1} G_{P L L}\left(s-j \omega_{1}\right) G_{s v}\left(s-j 2 \omega_{1}\right) \tilde{u}_{i}\left(s-j 2 \omega_{1}\right) \\
+\left[2+V_{1} G_{P L L}\left(s-j \omega_{1}\right)\right] G_{s v}(s) \tilde{u}_{i}(s)-2 G_{s v}\left(s+j 2 \omega_{1}\right) \tilde{u}_{i}\left(s+j 2 \omega_{1}\right)\end{array}\right\}$ & $(27 . b)$ \\
\hline$f_{\theta u,-2}(s)=j G_{P L L}\left(s-j \omega_{1}\right) G_{s v}\left(s-j 2 \omega_{1}\right)\left[2+V_{1} G_{P L L}\left(s+j \omega_{1}\right)\right] /[2 \Delta(s)]$ & $(30 . \mathrm{a})$ \\
\hline$f_{\theta u, 0}(s)=-j G_{s v}(s)\left[G_{P L L}\left(s-j \omega_{1}\right)+G_{P L L}\left(s+j \omega_{1}\right)+V_{1} G_{P L L}\left(s-j \omega_{1}\right) G_{P L L}\left(s+j \omega_{1}\right)\right] / \Delta(s)$ & (30.b) \\
\hline$f_{\theta u, 2}(s)=j G_{P L L}\left(s+j \omega_{1}\right) G_{s v}\left(s+j 2 \omega_{1}\right)\left[2+V_{1} G_{P L L}\left(s-j \omega_{1}\right)\right] /[2 \Delta(s)]$ & (30.c) \\
\hline $\begin{aligned} \tilde{I}_{1}^{*}\left(s-j \omega_{1}\right)+\tilde{I}_{1}^{*}\left(s+j \omega_{1}\right) & =f_{l u,-2}(s) \tilde{u}_{i}\left(s-j 2 \omega_{1}\right)+f_{l u, 0}(s) \tilde{u}_{i}(s)+f_{l u, 2}(s) \tilde{u}_{i}\left(s+j 2 \omega_{1}\right) \\
& +f_{l i,-2}(s) \tilde{i}_{g}\left(s-j 2 \omega_{1}\right)+f_{l i, 0}(s) \tilde{i}_{g}(s)+f_{l i, 2}(s) \tilde{g}_{g}\left(s+j 2 \omega_{1}\right)\end{aligned}$ & $(31)$ \\
\hline$f_{I i,-2}(s)=G_{L}\left(s-j \omega_{1}\right) H_{v}\left(s-j \omega_{1}\right)\left[V_{1}-\left(s-j 2 \omega_{1}\right) L I_{1} e^{j \varphi_{i 1}}-j \omega_{1} L I_{1} e^{j \varphi_{i}}\right]$ & $(33 . a)$ \\
\hline$f_{I i, 0}(s)=G_{L}\left(s+j \omega_{1}\right) H_{v}\left(s+j \omega_{1}\right)\left[V_{1}-s L I_{1} e^{j \varphi_{11}}-j \omega_{1} L I_{1} e^{j \varphi_{1}}\right]+G_{L}\left(s-j \omega_{1}\right) H_{v}\left(s-j \omega_{1}\right)\left[V_{1}-s L I_{1} e^{-j \varphi_{11}}+j \omega_{1} L I_{1} e^{-j \varphi_{i 1}}\right]$ & (33.b) \\
\hline$f_{I i, 2}(s)=G_{L}\left(s+j \omega_{1}\right) H_{v}\left(s+j \omega_{1}\right)\left[V_{1}-\left(s+j 2 \omega_{1}\right) L I_{1} e^{-j \varphi_{i 1}}+j \omega_{1} L I_{1} e^{-j \varphi_{11}}\right]$ & (33.c) \\
\hline$)=\frac{2 L s+2 G_{d}(s) P I_{3}(s) G_{s i}(s)-G_{d}(s) P I_{3}(s) f_{I i, 0}(s)}{2-2 G_{d}(s) G_{s v}(s)+j I_{1} G_{d}(s) P I_{3}(s) f_{\theta u, 0}(s)+G_{d}(s) P I_{3}(s) f_{L u, 0}(s)}$ & (37) \\
\hline
\end{tabular}

Substituting (34) into (35) gives

$$
\begin{aligned}
& {\left[L s+G_{d}(s) P I_{3}(s) G_{s i}(s)\right] \tilde{i}_{g}(s)} \\
& =\left[1-G_{d}(s) G_{s v}(s)\right] \tilde{u}_{i}(s)+G_{d}(s) P I_{3}(s) \tilde{i}_{g_{-} r e f}(s)
\end{aligned}
$$

Substituting (25) into (36), the input impedance which only considers the fundamental perturbed components is obtained in (37) shown at the bottom of the page. In order to include the interactions between different domains, frequency-shifting (36)

$$
\frac{C_{d c}}{2} \frac{d \tilde{x}_{u}(t)}{d t}+\frac{\tilde{x}_{u}(t)}{R_{d c}}=i_{g}^{*}(t) \tilde{u}_{i}(t)+u_{i}^{*}(t) \tilde{i}_{g}(t)-L i_{g}^{*}(t) \frac{d \tilde{r}_{g}(t)}{d t}-L \frac{d i_{g}^{*}(t)}{d t} \tilde{i}_{g}(t)
$$




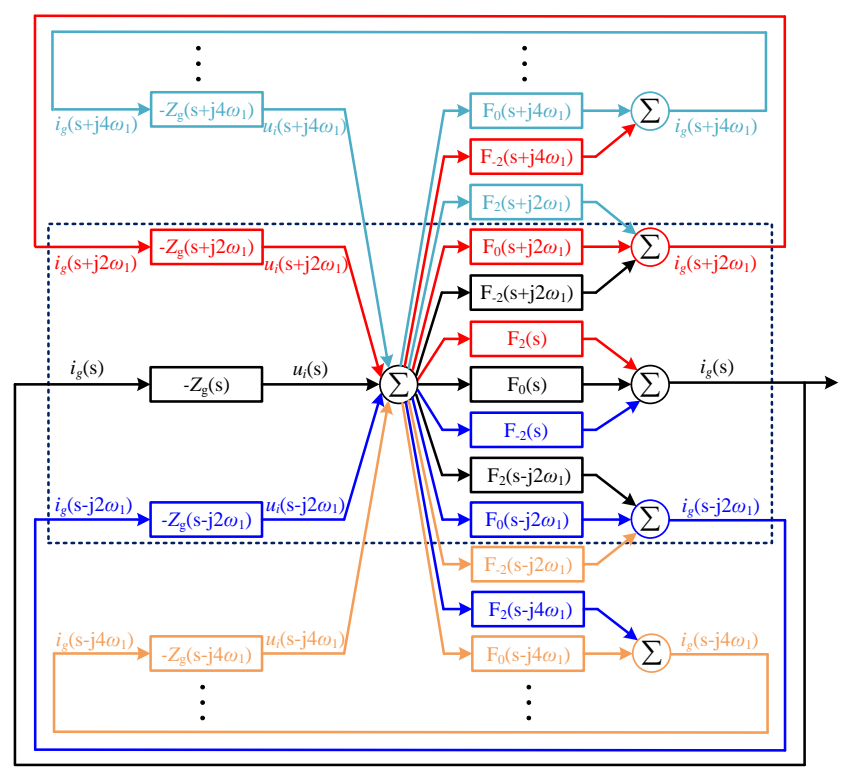

Fig. 2. Multi-frequency model diagram of the single-phase voltage source rectifier considering the grid impedance.

to $\omega \pm 2 \omega_{1}$, and then the perturbed current at frequencies $\omega \pm 2 \omega_{1}$ are solved as

$$
\begin{gathered}
\tilde{i}_{g}\left(s-j 2 \omega_{1}\right)=f_{x 1}(s) \tilde{u}_{i}\left(s-j 2 \omega_{1}\right) \\
+f_{x 2}(s) \tilde{u}_{i}(s)+f_{x 3}(s) \tilde{i}_{g}(s) \\
\tilde{i}_{g}\left(s+j 2 \omega_{1}\right)=f_{y 1}(s) \tilde{u}_{i}\left(s+j 2 \omega_{1}\right) \\
+f_{y 2}(s) \tilde{u}_{i}(s)+f_{y 3}(s) \tilde{i}_{g}(s)
\end{gathered}
$$

where the harmonic components with quadruple line frequency deviation are assumed to be zero. And $f_{x 1}(s), f_{x 2}(s), f_{x 3}(s), f_{y 1}(s)$, $f_{y 2}(s)$ and $f_{y 3}(s)$ are given as (40) and (41) shown at the bottom of the page.

Substituting (38) and (39) into (36), the harmonic current at frequency $\omega$ is provided as

$$
\begin{aligned}
\tilde{i}_{g}(s) & =F_{-2}(s) \tilde{u}_{i}\left(s-j 2 \omega_{1}\right)+F_{0}(s) \tilde{u}_{i}(s) \\
& +F_{2}(s) \tilde{u}_{i}\left(s+j 2 \omega_{1}\right)
\end{aligned}
$$

where $F_{-2}(\mathrm{~s}), F_{0}(\mathrm{~s})$ and $F_{2}(\mathrm{~s})$ are given as (43) shown at the bottom of the page.

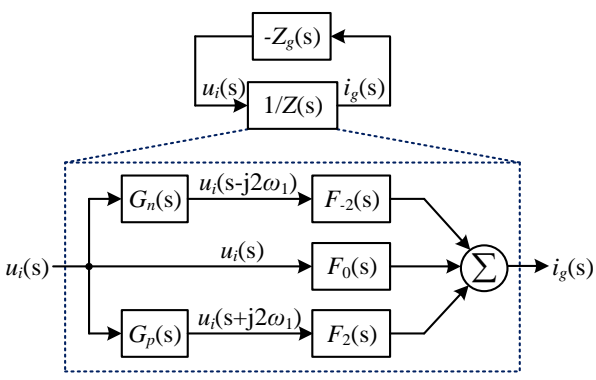

Fig. 3. Single-phase voltage source rectifier model diagram with embedded frequency-coupling effects.

If the effect of grid impedance is neglected, the input impedance is

$$
Z_{o p}(s)=1 / F_{0}(s) \text {. }
$$

However, due to the presence of grid impedance, the harmonic currents would generate the voltage at the same frequency and thus constitute additional feedback loops, which are constructed as Fig. 2. It is observed that the order of harmonic would extend to infinite due to the interactions between the FCE and the grid impedance.

Due to the low-pass characteristics of the system, only the components at frequencies $\omega$ and $\omega \pm 2 \omega_{1}$ are considered here, as denoted by the dashed block in Fig. 2. The harmonic voltage at frequencies $\omega \pm 2 \omega_{1}$ are derived as

$$
\begin{aligned}
& \tilde{u}_{i}\left(s-j 2 \omega_{1}\right)=G_{n}(s) \tilde{u}_{i}(s) \\
& \tilde{u}_{i}\left(s+j 2 \omega_{1}\right)=G_{p}(s) \tilde{u}_{i}(s)
\end{aligned}
$$

where

$$
\begin{aligned}
G_{n}(s) & =-\frac{F_{2}\left(s-j 2 \omega_{1}\right)}{Y_{g}\left(s-j 2 \omega_{1}\right)+F_{0}\left(s-j 2 \omega_{1}\right)} \\
G_{p}(s) & =-\frac{F_{-2}\left(s+j 2 \omega_{1}\right)}{Y_{g}\left(s+j 2 \omega_{1}\right)+F_{0}\left(s+j 2 \omega_{1}\right)} .
\end{aligned}
$$

And $Y_{g}(s)$ represents the grid admittance, i.e., $Y_{g}(s)=1 / Z_{g}(s)$. Thus, the single-frequency model at the analyzed frequency $\omega$ can be depicted as Fig. 3.

$$
\begin{aligned}
& f_{x 1}(s)=\left\{2-2 G_{d}\left(s-j 2 \omega_{1}\right) G_{s v}\left(s-j 2 \omega_{1}\right)+G_{d}\left(s-j 2 \omega_{1}\right) P I_{3}\left(s-j 2 \omega_{1}\right)\left[j I_{1} f_{\theta u, 0}\left(s-j 2 \omega_{1}\right)+f_{I u, 0}\left(s-j 2 \omega_{1}\right)\right]\right\} /\left[2 \Delta_{x}(s)\right] \quad(40 . \mathrm{a}) \\
& f_{x 2}(s)=G_{d}\left(s-j 2 \omega_{1}\right) P I_{3}\left(s-j 2 \omega_{1}\right)\left[j I_{1} f_{\theta u, 2}\left(s-j 2 \omega_{1}\right)+f_{l u, 2}\left(s-j 2 \omega_{1}\right)\right] /\left[2 \Delta_{x}(s)\right] \\
& f_{x 3}(s)=G_{d}\left(s-j 2 \omega_{1}\right) P I_{3}\left(s-j 2 \omega_{1}\right) f_{I i, 2}\left(s-j 2 \omega_{1}\right) /\left[2 \Delta_{x}(s)\right] \\
& \Delta_{x}(s)=L\left(s-j 2 \omega_{1}\right)+G_{d}\left(s-j 2 \omega_{1}\right) P I_{3}\left(s-j 2 \omega_{1}\right)\left[G_{s i}\left(s-j 2 \omega_{1}\right)-\frac{1}{2} f_{I i, 0}\left(s-j 2 \omega_{1}\right)\right] \\
& f_{y 1}(s)=2-2 G_{d}\left(s+j 2 \omega_{1}\right) G_{s v}\left(s+j 2 \omega_{1}\right)+G_{d}\left(s+j 2 \omega_{1}\right) P I_{3}\left(s+j 2 \omega_{1}\right)\left[j I_{1} f_{\theta u, 0}\left(s+j 2 \omega_{1}\right)+f_{I u, 0}\left(s+j 2 \omega_{1}\right)\right] /\left[2 \Delta_{y}(s)\right] \\
& f_{y 2}(s)=G_{d}\left(s+j 2 \omega_{1}\right) P I_{3}\left(s+j 2 \omega_{1}\right)\left[j I_{1} f_{\theta u,-2}\left(s+j 2 \omega_{1}\right)+f_{I u,-2}\left(s+j 2 \omega_{1}\right)\right] /\left[2 \Delta_{y}(s)\right] \\
& f_{y 3}(s)=G_{d}\left(s+j 2 \omega_{1}\right) P I_{3}\left(s+j 2 \omega_{1}\right) f_{l i,-2}\left(s+j 2 \omega_{1}\right) /\left[2 \Delta_{y}(s)\right] \\
& \Delta_{y}(s)=L\left(s+j 2 \omega_{1}\right)+G_{d}\left(s+j 2 \omega_{1}\right) P I_{3}\left(s+j 2 \omega_{1}\right)\left[G_{s i}\left(s+j 2 \omega_{1}\right)-\frac{1}{2} f_{I i, 0}\left(s+j 2 \omega_{1}\right)\right] \\
& F_{-2}(s)=G_{d}(s) P I_{3}(s)\left[j I_{1} f_{\theta u,-2}(s)+f_{I u,-2}(s)+f_{I i,-2}(s) f_{x 1}(s)\right] /\left[2 \Delta_{F}(s)\right] \\
& F_{0}(s)=2-2 G_{d}(s) G_{s v}(s)+G_{d}(s) P I_{3}(s)\left[j I_{1} f_{\theta u, 0}(s)+f_{I u, 0}(s)+f_{I i,-2}(s) f_{x 2}(s)+f_{I i, 2}(s) f_{y 2}(s)\right] /\left[2 \Delta_{F}(s)\right] \\
& F_{2}(s)=G_{d}(s) P I_{3}(s)\left[j I_{1} f_{\theta u, 2}(s)+f_{I u, 2}(s)+f_{I i, 2}(s) f_{y 1}(s)\right] /\left[2 \Delta_{F}(s)\right] \\
& \Delta_{F}(s)=L s+G_{d}(s) P I_{3}(s) G_{s i}(s)-\frac{1}{2} G_{d}(s) P I_{3}(s)\left[f_{I i, 0}(s)+f_{I i,-2}(s) f_{x 3}(s)+f_{I i, 2}(s) f_{y 3}(s)\right]
\end{aligned}
$$



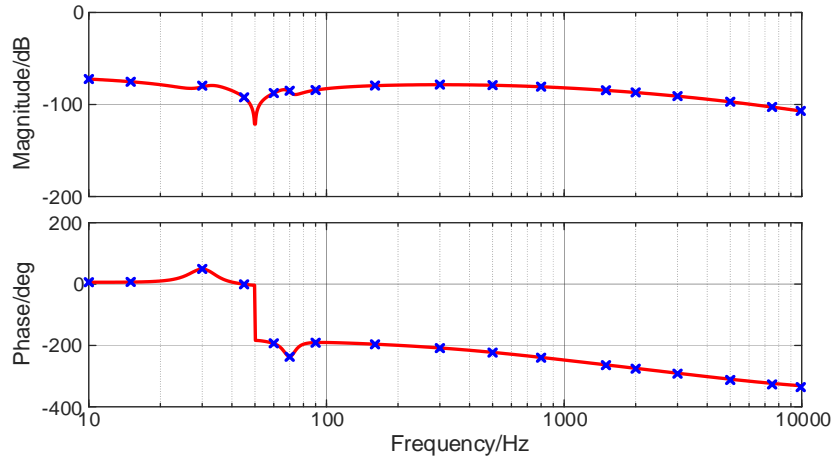

(a) $[\cos (\theta)](s) / \tilde{u}_{i}\left(s+j 2 \omega_{1}\right)$

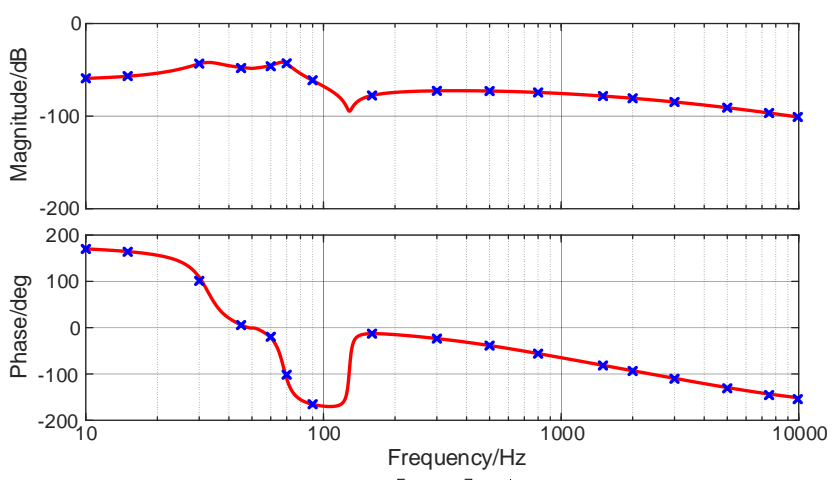

(b) $[\cos (\theta)](s) / \tilde{u}_{i}(s)$



(c) $[\cos (\theta)](s) / \tilde{u}_{i}\left(s-j 2 \omega_{1}\right)$

Fig. 4. Modeled and simulated response of $\cos (\theta)$. Solid lines: Model prediction; Dots: Numerical simulation results.

From Fig. 3, the single-frequency rectifier input impedance considering the FCE at a certain grid impedance is derived as

$$
Z(s)=\frac{1}{F_{0}(s)+F_{-2}(s) G_{n}(s)+F_{2}(s) G_{p}(s)} .
$$

The built impedance model $Z(\mathrm{~s})$ is the conventional SISO type impedance, such that the stability analysis of the system can be determined by Nyquist Criterion rather than GNC, which reduces the complexity of the analysis. And the built input impedance can be directly measured. Moreover, it is revealed that the rectifier input impedance is dependent on the grid impedance, which is neglected in most literatures. Besides, the proposed modeling approach, which only requires Laplace transform and frequency-shifting operation, avoids he complicated convolution calculation in the frequency domain.

\section{SIMULATION STUDY}

Simulations are performed in MATLAB/Simulink to verify the validity of the proposed models. The specifications of this system are summarized in Table I.

TABLE I

THE SIMULINK PARAMETERS OF THE SYSTEM

\begin{tabular}{cll}
\hline \hline Symbol & \multicolumn{1}{c}{ Description } & \multicolumn{1}{c}{ Value } \\
\hline$u_{g}$ & Grid voltage & $100 \mathrm{~V}(\mathrm{rms})$ \\
$\omega_{1}$ & Input angular frequency & $100 \pi \mathrm{rad} / \mathrm{s}$ \\
$L$ & Input filter inductance & $2 \mathrm{mH}$ \\
$C_{d c}$ & Output capacitance & $500 \mu \mathrm{F}$ \\
$R_{d c}$ & Load resistance & $60 \Omega$ \\
$\omega_{i} / \omega_{v}$ & ADC cutoff frequency & $9000 \pi \mathrm{rad} / \mathrm{s}$ \\
$k_{p 1}$ & Proportional coefficient of the PLL & 2.7207 \\
$k_{i 1}$ & Integral coefficient of the PLL & 49.348 \\
$k_{p 2}$ & Proportional coefficient of the & 0.0005 \\
& voltage regulator & \\
$k_{i 2}$ & Integral coefficient of the voltage & 0.008 \\
& regulator & 14.215 \\
$k_{p 3}$ & Proportional coefficient of the & \\
& current regulator & 50532.37 \\
$k_{i 3}$ & Integral coefficient of the current & $200 \pi$ \\
$\omega_{n}$ & regulator & Notch filter param. \\
$\sigma$ & Notch filter param. & 4737.4 \\
$\tau$ & Low-pass filter param. & 0.00398 \\
$u_{d c}^{*}$ & Output voltage reference & $200 \mathrm{~V}$ \\
$T_{s}$ & Control period & $50 \mu \mathrm{s}$ \\
\hline & &
\end{tabular}

In order to validate the small-signal model of PLL, the $s$ domain expression of linearized synchronous signal is given as

$$
[\cos (\theta)](s)=\frac{j}{2}\left[\begin{array}{l}
f_{\theta u, 2}(s) \tilde{u}_{i}\left(s+j 2 \omega_{1}\right)+f_{\theta u, 0}(s) \tilde{u}_{i}(s) \\
+f_{\theta u,-2}(s) \tilde{u}_{i}\left(s-j 2 \omega_{1}\right)
\end{array}\right] .
$$

The modeled and simulated frequency response from perturbed voltage at different frequencies to synchronous signal at frequency $\omega$ are plotted in Fig. 4. The results show that the simulated response is matched well with the modeled response, which verifies the validity of the small-signal model of PLL.

The impedance measurement scheme is depicted as Fig. 5. A perturbation voltage $u_{p}$ is injected, which excites the perturbations of corresponding frequency on the PCC voltage and input current. Then the FFT is applied to the PCC voltage and input current for impedance calculation. In this scheme, the influence of grid impedance is taken into consideration. The frequency response characteristics of the built impedance models and its corresponding simulation results under different grid impedances are plotted as shown in Fig. 6. As observed, the impedance simulation results are in good accordance with $Z(\mathrm{~s})$, which validates the proposed impedance modeling approach is accurate. The impedance $Z_{c}(s)$ which only considers the fundamental perturbed components has a large deviation in the regions around the fundamental frequency. The $Z_{o p}(s)$ achieves higher accuracy compared with $Z_{c}(s)$, but the accuracy will degrade as the grid impedance increases due to the neglect of grid impedance. It is suggested that the interactions between grid impedance and FCE should be considered in the input impedance modeling of single-phase VSR. 


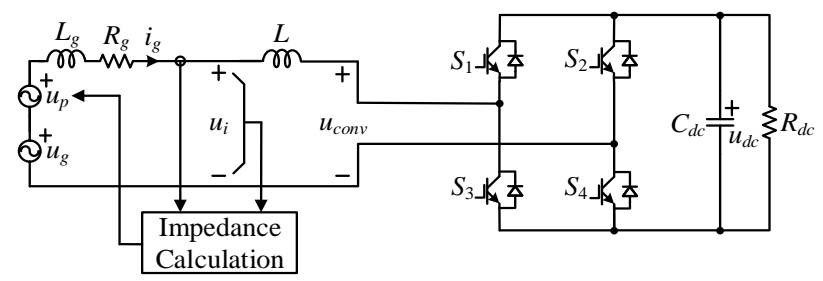

Fig. 5. Impedance measurement scheme for single-phase converters.
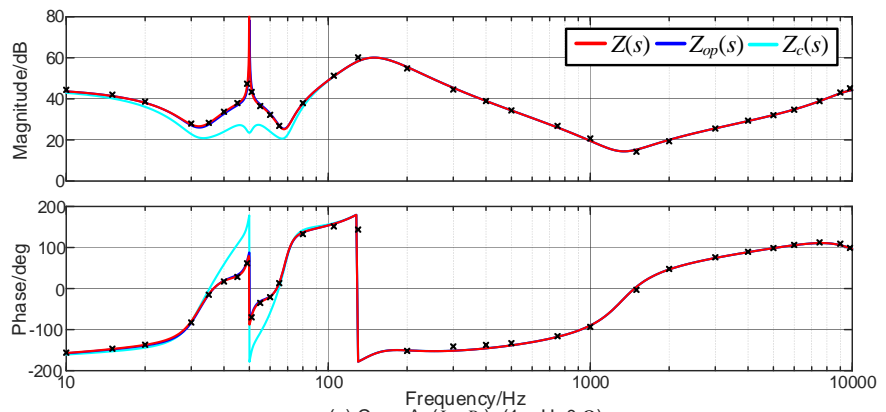

(a) Case A: $\left(L_{g}, R_{g}\right)=(1 \mathrm{mH}, 0 \Omega)$


Fig. 6. Impedance responses. Solid lines: Model prediction; Dots: Numerical simulation results.

\section{CONCLUSION}

In this paper, the single-frequency input impedance modeling of the single-phase voltage source rectifiers is presented, which considers the frequency-coupling effect. It is found that the established input impedance varies along with the grid impedance. In the proposed modeling method, only Laplace transform and frequency-shifting operation are required, avoiding the complicated convolution calculation in the frequency domain. In addition, the modeling method is also applicable to other power converters

\section{ACKNOWLEDGMENT}

This work is supported by National Natural Science Foundation of China under Grant 61933011.

\section{REFERENCES}

[1] H. Hu, H. Tao, F. Blaabjerg, X. Wang, Z. He and S. Gao, "Train-network interactions and stability evaluation in high-speed railways-part I: phenomena and modeling," IEEE Trans. Power Electron., vol. 33, no. 6, pp. 4627-4642, Jun. 2018.

[2] X. Wang, F. Blaabjerg and W. Wu, "Modeling and analysis of harmonic stability in an ac power-electronics-based power system," IEEE Trans. Power Electron., vol. 29, no. 12, pp. 6421-6432, Dec. 2014.

[3] J. Sun, G. Wang, X. Du and H. Wang, "A theory for harmonics created by resonance in converter-grid systems," IEEE Trans. Power Electron., vol. 34, no. 4, pp. 3025-3029, Apr. 2019.

[4] V. Salis, A. Costabeber, S. M. Cox, F. Tardelli, and P. Zanchetta, "Experimental validation of harmonic impedance measurement and LTP Nyquist criterion for stability analysis in power converter networks," IEEE Trans. Power Electron., vol. 34, no. 8, pp. 7972-7980, Aug. 2019.

[5] J. Sun, "Small-signal methods for ac distributed power systems-a review," IEEE Trans. Power Electron., vol. 24, no. 11, pp. 2545-2554, Nov. 2009.

[6] X. Wang and F. Blaabjerg, "Harmonic stability in power electronic-based power systems: concept, modeling, and analysis," IEEE Trans. Smart Grid, vol. 10, no. 3, pp. 2858-2870, May 2019.

[7] N. M. Wereley, "Analysis and control of linear periodically time varying systems," Ph.D. dissertation, Dept. of Aeronautics and Astronautics, MIT Inst. Technol., 1991.

[8] E. Mollerstedt, "Dynamic analysis of harmonics in electrical systems," Ph.D. dissertation, Dept. Automatic Control, Lund Inst. Technol., Lund, Sweden, 2000.

[9] H. Wang, M. Wu and J. Sun, "Analysis of low-frequency oscillation in electric railways based on small-signal modeling of vehicle-grid systems in dq frame," IEEE Trans. Power Electron., vol. 30, no. 9, pp. 5318-5330, Sep. 2015.

[10] S. Lissandron, L. Santa, P. Mattavelli and B. Wen, "Experimental validation for impedance-based small-signal stability analysis of singlephase interconnected power systems with grid-feeding inverters," IEEE J. Emerg. Sel. Topics Power Electron., vol. 4, no. 1, pp. 103-115, Mar. 2016.

[11] J. Sun and K. Karimi, "Small-signal input impedance modeling of linefrequency rectifiers," IEEE Trans. Aerosp. Electron. Syst., vol. 44, no. 4, pp. 1489-1497, Oct. 2008.

[12] H. Zhang, Z. Liu, S. Wu and Z. Li, "Input impedance modeling and verification of single-phase voltage source converters based on harmonic linearization," IEEE Trans. Power Electron., vol. 34, no. 9, pp. 8544-8554, Sep. 2019.

[13] Q. Qian, S. Xie, J. Xu, K. Xu, S. Bian and N. Zhong, "Output impedance modeling of single-phase grid-tied inverters with capturing the frequencycoupling effect of PLL," IEEE Trans. Power Electron., early access, 2019. 